\title{
Physiological and Subjective Responses to Low Relative Humidity
}

\author{
Yujin Sunwoo ${ }^{1)}$, Chinmei Chou ${ }^{3)}$, Junko Takeshita ${ }^{1)}$, Motoko Murakami ${ }^{2)}$ \\ and Yutaka Tochihara ${ }^{3)}$ \\ 1) Department of Ergonomics, Kyushu Institute of Design \\ 2) Kanebo Cosmetics Inc. \\ 3) Department of Ergonomics, Faculty of Design, Kyushu University
}

\begin{abstract}
In order to investigate the influence of low relative humidity, we measured saccharin clearance time (SCT), frequency of blinking, heart rate (HR), blood pressure, hydration state of skin, transepidermal water loss (TEWL), recovery sebum level and skin temperature as physiological responses. We asked subjects to judge thermal, dryness and comfort sensations as subjective responses using a rating scale. Sixteen non-smoking healthy male students were selected. The pre-room conditions were maintained at an air temperature (Ta) of $25^{\circ} \mathrm{C}$ and a relative humidity (RH) of $50 \%$. The test room conditions were adjusted to provide a Ta of $25^{\circ} \mathrm{C}$ and $\mathrm{RH}$ levels of $10 \%, 30 \%$ and $50 \%$.

RH had no effect on the activity of the sebaceous gland and on cardiovascular reactions like blood pressure and HR. However, it was obvious that low RH affects SCT, the dryness of the ocular mucosa and the stratum corneum of the skin and causes a decrease in mean skin temperature. Under $30 \% \mathrm{RH}$, the eyes and skin become dry, and under $10 \% \mathrm{RH}$ the nasal mucous membrane becomes dry as well as the eyes and skin, and the mean skin temperature decreases. These findings suggested that to avoid dryness of the eyes and skin, it is necessary to maintain an RH greater than $30 \%$, and to avoid dryness of the nasal mucous membrane, it is necessary to maintain an RH greater than $10 \%$. Subjects felt cold immediately after a change in RH while they had only a slight perception of dryness at the change of humidity. $J$ Physiol Anthropol 25(1): 7-14, 2006 http://www.jstage.jst.go.jp/ browse/jpa2
\end{abstract}

[DOI: $10.2114 / j p a 2.25 .7]$

Keywords: low relative humidity, saccharin clearance time, frequency of blinking, skin physiology, subjective responses

\section{Introduction}

In winter, indoor dryness due to heating causes many health problems. There are several regulations regarding the lowest limit for indoor humidity. In Japan, the "Law for Maintenance of Sanitation in Buildings" states that the indoor relative humidity (RH) should be kept at more than $40 \%$ (Ogawa, 1999), but several surveys reported that it is difficult to maintain this level (Koshimizu et al., 2002). In offices in winter, under a low RH environment, the skin becomes dry and itchy, and skin problems occur (Gaul et al., 1952). When the mucous membrane of the nose and throat becomes dry (Rankin, 1998), bacteria and virus can easily infect the human body. Moreover, the survival rates of the influenza virus increase under low RH (Harper, 1961, 1963), and the virus becomes very active in the air, thus causing influenza, common colds, and other respiratory infections (Satsuta et al., 1985). Therefore, in our daily lives, it is important to maintain the optimum indoor humidity level as well as the optimum room temperature.

The amount of inspired air is estimated at about 10,000-20,000 liters per day. Because so much air is inspired, the respiratory tract can be easily infiltrated by foreign bodies. The particles in the air adhere to the nasal mucous membrane, from which they can be removed by nasal blowing or expectorated as sputum after passing through the nasopharynx by means of mucociliary movement. Sometimes they pass through the oropharynx, laryngopharynx, and esophagus to the stomach and are digested. This mucociliary clearance is the fundamental self-defense mechanism by which the dust, bacteria and viruses in the inspired air are filtered in order to maintain health. The factors influencing the mucociliary function are high or low temperature and dry air. Many studies have been carried out to investigate the effect of low RH on the human body, including the effect on skin dryness (Yoshikuni et al., 1985) and dryness of the eyes (Eng et al., 1982 ; Nilsson et al., 1986 ; Flynn et al., 1988), while very few studies on the activity of the upper respiratory tract mucous membrane have been conducted.

Therefore, this study aims to investigate how low RH caused by heating influences the human body during winter by measuring the mucociliary activity as well as the functions of the eyes and skin and the thermal, dryness and comfort sensations under $\mathrm{RH}$ of $50 \%, 30 \%$ and $10 \%$ at the same 
Table 1 Physical characteristics of subjects $(n=16)$

Values are Mean \pm S.D.

\begin{tabular}{cccc}
\hline Age & Height $(\mathrm{cm})$ & Body weight $(\mathrm{kg})$ & BMI \\
\hline $22.6 \pm 2.7$ & $174.5 \pm 4.5$ & $60.5 \pm 5.4$ & $19.8 \pm 1.0$ \\
\hline
\end{tabular}

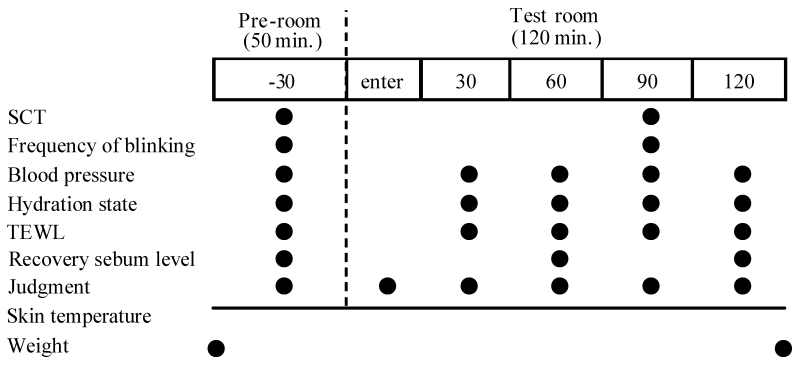

Fig. 1 Schedule for experiment.

temperature.

\section{Methods}

\section{Subjects}

Sixteen non-smoking healthy male students were selected. The means and standard variations of their age, height, body weight, and body mass index (BMI) are shown in Table 1. Written informed consent was obtained from all subjects after a full explanation of the experimental purpose and protocol.

\section{Procedures}

This study was conducted from February to March. The preroom conditions were maintained at an air temperature (Ta) of $25^{\circ} \mathrm{C}$ and a relative humidity $(\mathrm{RH})$ of $50 \%$. The test room conditions were adjusted to provide a Ta of $25^{\circ} \mathrm{C}$ and $\mathrm{RH}$ levels of $10 \%, 30 \%$ and $50 \%$. The subjects wore short pants, a long-sleeved sweat shirt and trousers $(0.8 \mathrm{clo})$ in the pre-room. After waiting for $50 \mathrm{~min}$ in a sitting position in the pre-room, the subjects moved to the test room and sat on a chair for 120 min. Figure 1 shows the schedule for the experiment.

\section{Measurements}

Saccharin clearance time (SCT), frequency of blinking, heart rate (HR), blood pressure, hydration state of skin, transepidermal water loss (TEWL), recovery sebum level and skin temperatures were measured as physiological responses. Also, we asked the subjects to judge the thermal, dryness and comfort sensations with subjective responses using a rating scale.

Mucociliary transport was measured by SCT. A saccharin tablet $(2.5 \mathrm{~mm} \times 0.5 \mathrm{~mm})$ was placed just behind the anterior end of the nasal septum on the level of the anterior end of the middle nasal concha. The subjects were asked to sit quietly with their heads forward and not to sniff or sneeze. The time was measured beginning at the first perception of the sweet taste.
Table 2 The scale of subjective judgments

\begin{tabular}{|c|c|c|c|}
\hline & $\begin{array}{l}\text { Thermal } \\
\text { sensation }\end{array}$ & $\begin{array}{c}\text { Sensation of } \\
\text { dryness }\end{array}$ & $\begin{array}{l}\text { Thermal } \\
\text { comfort }\end{array}$ \\
\hline 3 & hot & extremely dry & very comfortable \\
\hline 2 & warm & dry & comfortable \\
\hline 1 & slightly warm & slightly dry & slightly comfortable \\
\hline 0 & neutral & neutral & neutral \\
\hline-1 & slightly cool & slightly humid & slightly uncomfortable \\
\hline-2 & cool & humid & uncomfortable \\
\hline-3 & cold & wet & extremely uncomfortable \\
\hline
\end{tabular}

Frequency of blinking was measured while the subjects counted the white spots flickering randomly on the center of the monitor for two minutes (each white spot was illuminated for one sec., and the interval between the illumination of the spots was $0.2-1.4 \mathrm{sec}$.). Blood pressure and HR were checked at the right upper arm using an automatic tonometer (HEM737, OMRON, JAPAN) every $30 \mathrm{~min}$. Both SCT and frequency of blinking were measured in the pre-room and $90 \mathrm{~min}$ after entering the test room.

The hydration state of the skin was obtained using the CORNEOMETER ${ }^{\circledR}$ CM825 (Courage+Khazaka electronic GmbH, GERMANY) and TEWL was obtained using the VapoMeter (Keystone Scientific, JAPAN). Both hydration state and TEWL were measured in the pre-room and in the test room on the right-side of the cheek and the back of the right hand three times every $30 \mathrm{~min}$.

The recovery sebum level was obtained using the SEBUMETER ${ }^{\circledR}$ SM 810 (Courage+Khazaka electronic $\mathrm{GmbH}$, GERMANY). It was measured in the pre-room after the removal of sebum using an alcohol sponge and in the test room every $60 \mathrm{~min}$ on the left-side of the cheek and the back of the left hand. Skin temperatures at eight local body sites (i.e., the forehead, chest, shoulder, forearm, abdomen, hip, thigh, and foot) were measured with thermistors every minute.

Mean skin temperature was calculated by applying Fukuda's 12-point method (Watanabe, 1976), i.e. (forehead $\times 9.8+$ chest $\times 8.3+$ shoulder $\times 8.3+$ forearm $\times 19.6+$ abdomen $\times 8.1+$ hi $\mathrm{p} \times 8.1+$ thigh $\times 30.6+$ foot $\times 7.2) / 100=\overline{\mathrm{T}}_{\mathrm{SK}}$.

Body weight loss was obtained by deducting the weight before the experiment from the weight at the end of the experiment.

Thermal, dryness and comfort sensations were evaluated once in the pre-room, once upon entering the test room, and then every $30 \mathrm{~min}$ thereafter. Subjects evaluated the thermal sensation for the head, trunk, legs and the whole body, and they evaluated the sensation of dryness for the nose, throat, eyes, face and hands. Table 2 shows the scales used for the subjective judgments.

\section{Statistical analysis}

Data for comparing the pre-room with the test room were analyzed by the Paired t-test. Results of the physiological and subjective data were analyzed by repeated-measure analysis of 


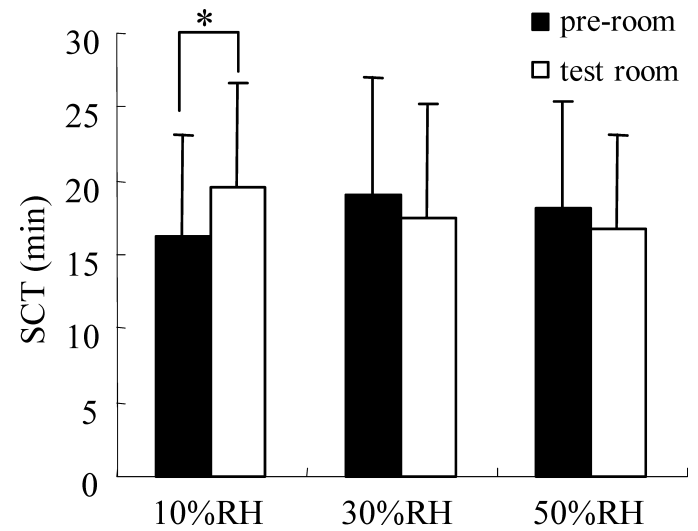

Fig. 2 Saccharin clearance time in the pre-room and $90 \mathrm{~min}$ after entering the test room. Values are means \pm S.D for all 16 subjects. ${ }^{*} p<0.05$ indicates significant differences between pre-room and test room values.

variance (ANOVA) using Visual State for Windows Release 4.5J Software (Stat Soft, Inc.).

The factors were conditions and time. A multiple comparison was performed using Scheffe. The relationship between SCT and hydration state was analyzed using Pearson's correlation coefficient test. Differences at $p<0.05$ were significant for all statistical analyses.

\section{Results}

\section{Physiological responses}

Figure 2 shows SCT in the pre-room and $90 \mathrm{~min}$ after entering the test room. SCT in $10 \% \mathrm{RH}$ increased meaningfully $(p<0.05)$ in comparison with SCT in the preroom, the difference being $3.35 \mathrm{~min}$., whereas the SCT values in $30 \% \mathrm{RH}$ and $50 \% \mathrm{RH}$ showed no significant differences.

Figure 3 shows the frequency of blinking in the pre-room and $90 \mathrm{~min}$ after entering the test room. Significant $(p<0.05)$ changes were observed in $30 \% \mathrm{RH}$ and $10 \% \mathrm{RH}$ in comparison with the pre-room level. Concerning heart rate, diastolic pressure and systolic pressure showed no significant change among humidity levels.

Figure 4 shows the hydration state of the skin. The hydration state of the face showed significant $(p<0.05)$ differences among humidity levels. Moreover, it was significantly $(p<0.001)$ affected by the interaction of humidity and time. Under RH levels of $10 \%$ and $30 \%$, the hydration state of the face decreased beginning at $30 \mathrm{~min}$ after entering the test room. However, at 60,90 , and 120 min the hydration state did not decrease any further and was stabilized. The hydration state of the hand showed significant $(p<0.001)$ differences at each time-point but no significant differences among humidity levels.

Figure 5 shows the relationship between SCT and the hydration state of the face under $10 \% \mathrm{RH}$. There was a significant $(p<0.05, r=-0.61)$ negative correlation between $\mathrm{SCT}$ and the hydration state of the face in $10 \% \mathrm{RH}$, showing

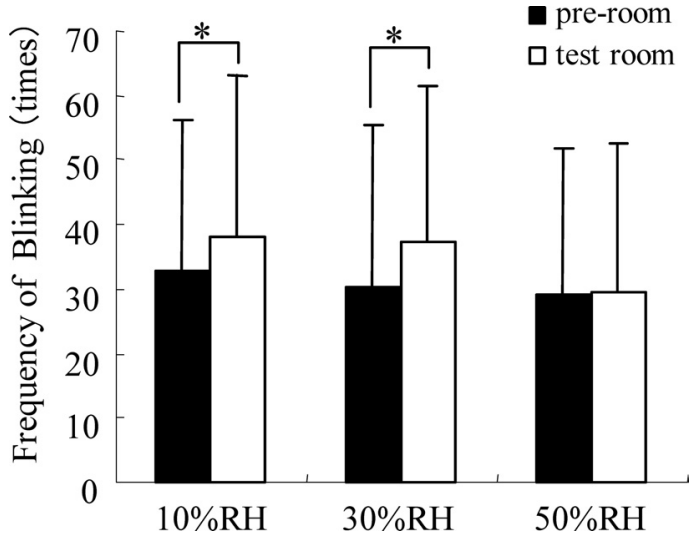

Fig. 3 Frequency of blinking in the pre-room and $90 \mathrm{~min}$ after entering the test room. Values are means \pm S.D for all 16 subjects. ${ }^{*} p<0.05$ indicates significant differences between pre-room and test room values.
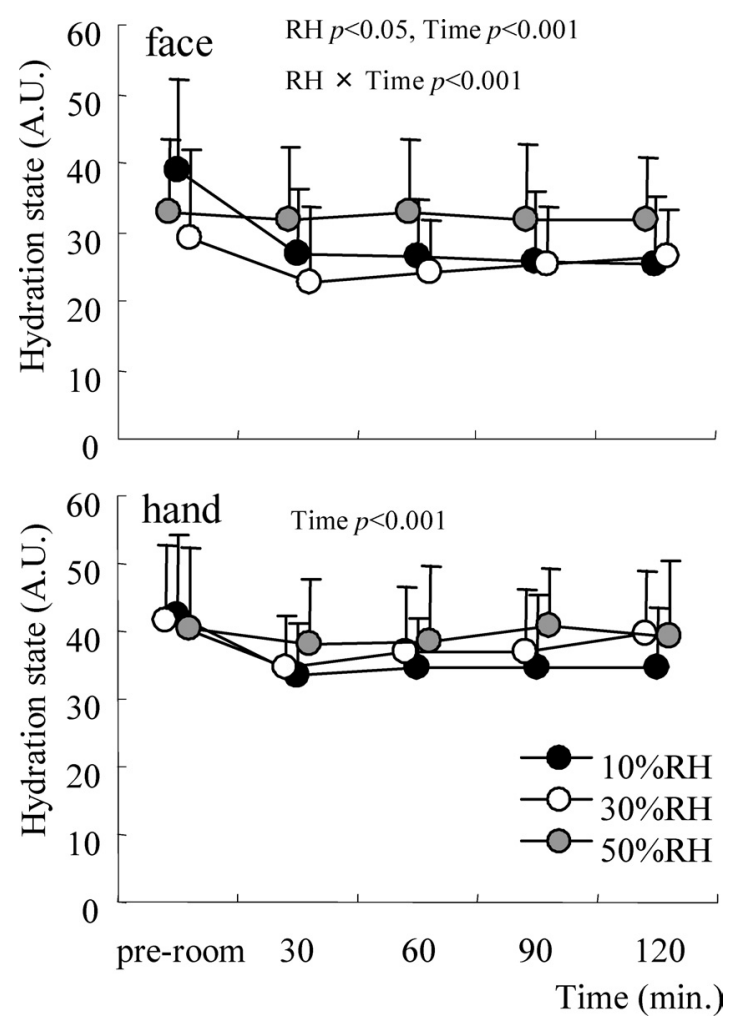

Fig. 4 Hydration state on the cheek and the hand. Values are means \pm S.D for all 16 subjects. Significant interactions (humidity $\times$ time) were determined by ANOVA.

that as the SCT became longer, the hydration state of the face became lower. But, under RH levels of $30 \%$ and $50 \%$, there was nosignificant correlation between SCT and the hydration state of the face.

Figure 6 shows the TEWL of the skin. The TEWL of the face showed significant $(p<0.05)$ differences among humidity levels. Moreover, it was significantly $(p<0.001)$ affected by the interaction of humidity and time. Under RH levels of $10 \%$ and 


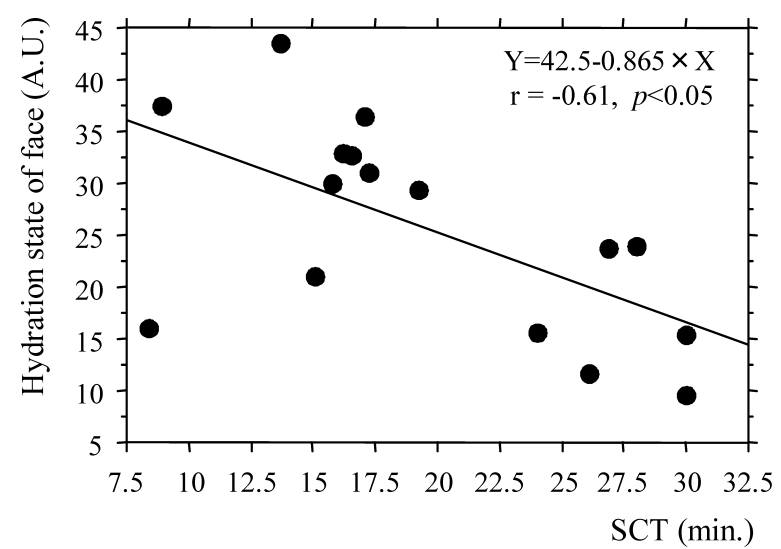

Fig. 5 Relationship between saccharin clearance time and hydration state on the cheek in $10 \% \mathrm{RH}$.
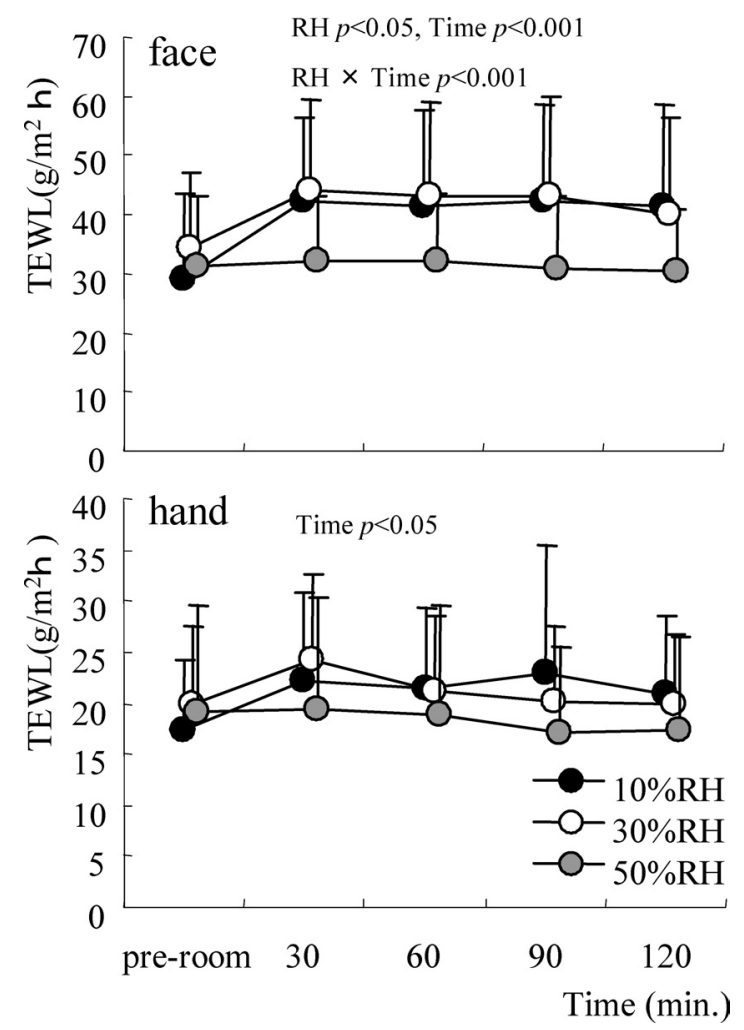

Fig. 6 Transepidermal water loss on the cheek and the hand. Values are means \pm S.D for all 16 subjects. Significant interactions (humidity $\times$ time) were determined by ANOVA.

$30 \%$, the TEWL of the face increased beginning at $30 \mathrm{~min}$ after entering the test room. However, at 60,90 , and $120 \mathrm{~min}$ the TEWL did not increase any further and was stabilized. The TEWL of the hand showed significant $(p<0.05)$ differences at each time-point but no significant differences among humidity levels. The recovery sebum level in the skin increased significantly $(p<0.001)$ as time passed, but there was no significant difference among humidity levels.

Figure 7 shows the change in mean skin temperature. The

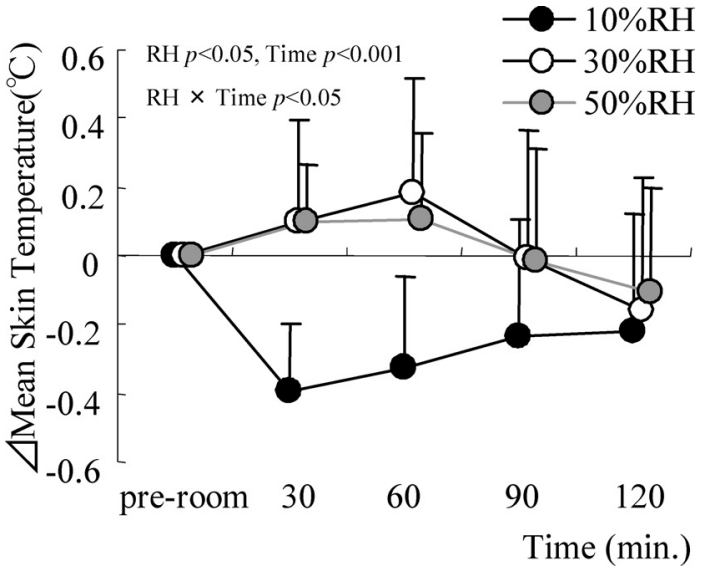

Fig. 7 Change of mean skin temperature. Values are means \pm S.D for all 16 subjects. Significant interactions (humidity $\times$ time) were determined by ANOVA.

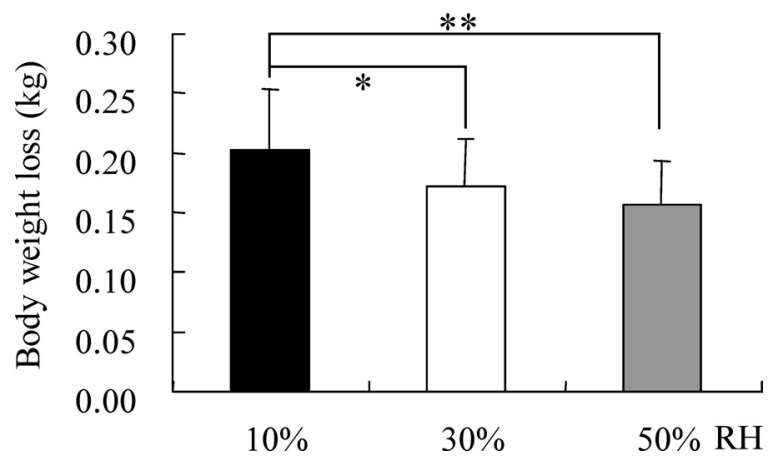

Fig. 8 Body weight loss. Values are means \pm S.D for all 16 subjects. ${ }^{*} p<0.05,{ }^{*} p<0.001$ Indicates a significant main effect (humidity) as determined by ANOVA.

change at each humidity level was significant $(p<0.05)$ showing the degree of decrease in mean skin temperature to be greater under $10 \% \mathrm{RH}$ than $50 \% \mathrm{RH}$.

Figure 8 shows body weight loss. There were significant $(p<0.001)$ differences among humidity levels showing a greater loss of body weight under $10 \% \mathrm{RH}$ than under $30 \%$ RH and $50 \%$ RH.

\section{Subjective responses}

Figure 9 shows the thermal sensation for each site and for the whole body on entering the test room and 90 min after entering the test room. For the thermal sensation observed just after moving to the test room from the pre-room, there were significant $(p<0.001)$ differences among humidity levels at the tested body sites except the head, showing that there was a subjective cooler sensation as the humidity decreased. Thermal sensation after $90 \mathrm{~min}$, however there were no significant differences among humidity levels.

Figure 10 shows the sensation of dryness at each site on entering the test room and $90 \mathrm{~min}$ after entering the test room. Regarding the sensation of dryness just after moving to the test room from the pre-room, there were significant $(p<0.05)$ 


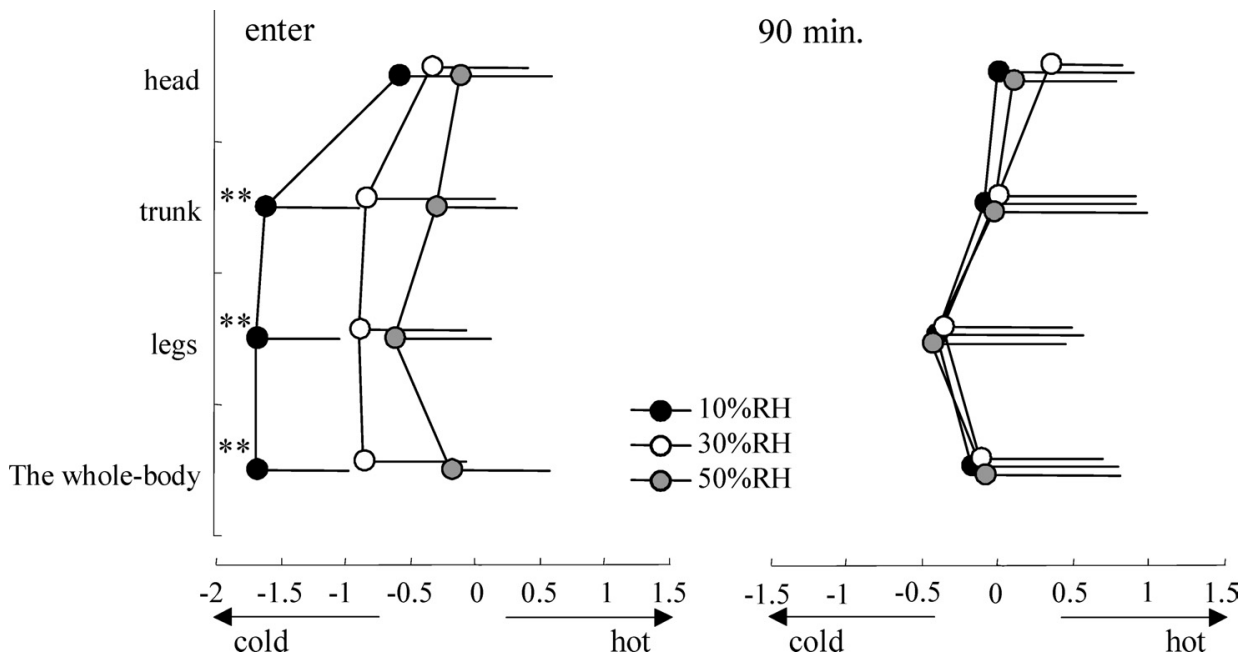

Fig. 9 Thermal sensation at each site and for the whole-body on entering the test room and 90 min after entering the test room. Values are means for all 16 subjects. ${ }^{*} p<0.001$ indicates a significant main effect (humidity) as determined by ANOVA.

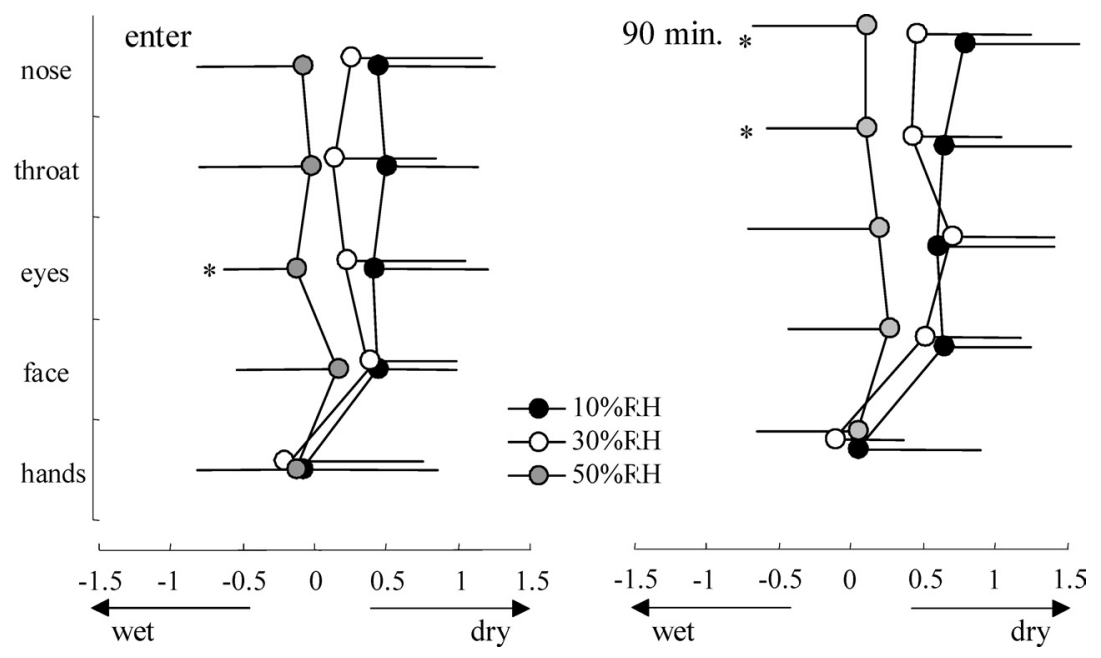

Fig. 10 Sensation of dryness at each site on entering the test room and $90 \mathrm{~min}$ after entering the test room. Values are means for all 16 subjects. ${ }^{*} p<0.05$ indicates a significant main effect (humidity) as determined by ANOVA.

differences among humidity levels only in the eye, showing that the subjective feeling of dryness increases as humidity decreases. After $90 \mathrm{~min}$, there were significant $(p<0.05)$ differences in the sensation of dryness in the nose and throat between each humidity level. There were no significant differences in thermal comfort among humidity levels.

\section{Discussion}

We investigated the effect of $10 \% \mathrm{RH}, 30 \% \mathrm{RH}$, and $50 \%$ RH on SCT, dryness of ocular mucosa, physiological function of the skin and cardiovascular reaction under an environmental temperature of $25^{\circ} \mathrm{C}$. In addition, we assessed the subjective thermal, dryness, and comfort sensations. In physiological responses, there was no effect of RH on the activity of the sebaceous gland or on cardiovascular reactions like blood pressure and HR. However, it was obvious that low RH affects
$\mathrm{SCT}$, dryness of the ocular mucosa and the stratum corneum of the skin and causes the mean skin temperature to decrease as a result of this dryness. SCT under 10\% RH increased more than it did in the pre-room with $50 \% \mathrm{RH}$. The frequency of blinking under $10 \% \mathrm{RH}$ and $30 \% \mathrm{RH}$ increased more than in the preroom with $50 \% \mathrm{RH}$. Under the low RH levels of $30 \%$ and $10 \%$, TEWL increased more than under $50 \% \mathrm{RH}$. As a result, it is obvious that the eyes and skin become dry in $30 \% \mathrm{RH}$, and the nasal cavity becomes dry as well as the eyes and skin in $10 \% \mathrm{RH}$.

Mucociliary clearance of the airway is an important selfdefense mechanism, and it plays an important role in the removal of foreign bodies. When this mechanism is impaired, the possibility of respiratory infections is increased. The saccharin method used in this experiment is widely used to assess the function of mucociliary clearance (Lale et al., 1998). An increase in SCT suggests a decrease in the activity of the 
mucociliary function. In this study, SCT increased significantly under the environment of $10 \% \mathrm{RH}$, suggesting that $10 \% \mathrm{RH}$ caused a decrease in the ciliary activity of the nasal mucous membrane (Fig. 2). Harper (1961) reported that the survival rate of the influenza virus is only $3-5 \%$ after 6 hours in an environment of $20.5-24^{\circ} \mathrm{C}$ and $50 \% \mathrm{RH}$ and above, but this rate increased to $66 \%$ under an environment of $20 \% \mathrm{RH}$. The influenza virus in the air loses infectivity in an environment with $50 \% \mathrm{RH}$ and above, but its survival time is prolonged in an environment with an RH below 50\% (Harper, 1963). Satsuta et al. (1985) reported that there is a seasonal factor in the rate of influenza infection, with respiratory infection prevalent especially in winter. This explains the long survival rate of the organism in environments with low humidity in winter, and the inflammation of the nasal and pharngolaryngeal mucosa caused by cold air inhalation makes it easier for these mucosa to be infiltrated by the organism. Therefore, according to our study, in an environment with an $\mathrm{RH}$ below $10 \%$, it is fairly easy to catch a respiratory infection caused by an influenza virus in the air due to the fact that dryness of the nasal mucocilia compromises the defense mechanism.

Frequency of blinking was measured to determine the effect of humidity on the mucous membrane of the eye in this experiment. The frequency of blinking seemed to reflect the state of dryness of the eye, because the blinking acts to supply moisture to the eye. In this experiment, the frequency of blinking increased significantly under environments of $10 \%$ $\mathrm{RH}$ and 30\% RH, suggesting that a low RH environment influences the activity of the mucous membrane of the eye (Fig. 3). An increase in the frequency of blinking is known to cause discomfort, while blinking is known to be greatly affected by psychological factors (Stern et al., 1984). In an experiment involving contact lens wearers (Nilsson et al., 1986), subjects in low RH environments (approximately less than $30 \%$ ) showed significantly shorter break-up times (BUT) on the lens and more prominent lens deposits than subjects in higher $\mathrm{RH}$ environments ( $\mathrm{RH}$ of more than 40\%). Many contact lens wearers in low RH environments complain about dry eyes and discomfort, whereas others seem to have no trouble at all. It was reported that for aircraft passengers and crew, low cabin humidity seemed to be the most significant factor contributing to discomfort for lens wearers (Eng et al., 1982). In recent years, as computer work has increased in office environments, problems related to the fatigability of eyes and psychological stress have emerged. In particular, because the RH in offices drops in winter due to excessive heating, an increase in the frequency of blinking occurs, and outbreaks of eye fatigue and stress are expected to result.

Grice et al. (1972) reported an increase in the TEWL of the skin as humidity decreases in the state of no perspiration. In our study, TEWL of the face increased significantly under $10 \%$ $\mathrm{RH}$ and $30 \% \mathrm{RH}$, which were both lower than the humidity level of the pre-room, and there was no change of TEWL under $50 \%$ RH, which was the same as the humidity level of the pre-room (Fig. 6). A marked decrease in the hydration state of the face was observed under 10\% RH and 30\% RH (Fig. 4). This decrease is thought to be the result of the loss of moisture from the stratum corneum due to the increased evaporation of moisture from the skin surface. The loss of body weight after the experiment grew as the level of humidity decreased (Fig. 8), suggesting that the evaporation of water from the body occurred in accord with the level of humidity. Moreover, it seems that a decrease of the mean skin temperature occurred under $10 \% \mathrm{RH}$ due to a loss of heat, which was caused by the evaporation of water from the body (Fig. 7). The recovery sebum level showed no significant difference among humidity levels. From this result involving the function of skin physiology, it was revealed that a change of $\mathrm{RH}$ affected parameters related to the moisture of the skin such as hydration state and TEWL, but there was no influence of humidity on sebum secretion. Cook et al. (1958) compared the texture of dry skin with that of non-dry skin according to the pattern of the skin surface. It is reported that the number of smaller peaks of crista cutis is reduced in dry skin. Also, Yoshikuni et al. (1985) concluded that decreases in the outdoor temperature and humidity cause cracking of the skin by decreasing the activity and water contents of the stratum corneum directly or indirectly. From our study it seems that dryness of the skin adversely affects the skin texture in the environment of $30 \%$ $\mathrm{RH}$ and below. And in addition to the change of the skin surface due to the decrease in moisture in the skin, outbreaks of dermatologic disorders like dry skin accompanied by itching occur.

Under $10 \% \mathrm{RH}$, a significant decrease in ciliary activity and hydration state of the skin were observed, and a significantly negative correlation between these parameters was also observed (Fig. 5). Under the environment of low RH in which the mucosa of the nasal cavity and the skin are apt to become dry, it was observed that dryness of the nasal mucosa became more severe as dryness of the skin became more severe. Therefore, it is thought that the response to the water loss that occurs under low RH is similar between the mucosa and the skin.

Maehara et al. (1991) reported that in a survey done in an airplane, the degree of dryness increased linearly as the $\mathrm{RH}$ in the environment decreased to below $40 \% \mathrm{RH}$, while with regard to thermal sensation, subjects tended to feel cool as $\mathrm{RH}$ decreased during the flight. Also, the subjects tended to feel warm when the average RH returned to $40-50 \%$ upon landing. In the present study, the subjects generally felt cool immediately after moving to the test room from the pre-room in 50\% RH at all RH levels. However, after $30 \mathrm{~min}$ their sensation of coolness was slight, and after $90 \mathrm{~min}$ there seemed to be no difference in thermal sensation at each RH level (Fig. 9). Humans seem to feel the change of humidity as a change of thermal sensation only momentarily at a certain temperature.

Regarding dryness in the skin, there were significant differences in dryness in the skin with changes in $\mathrm{RH}$ as measured physiologically, while in the subjective assessment 
the subjects felt no sense of dryness on the face and hands until after $90 \mathrm{~min}$ of $\mathrm{RH}$ change. In the case of the eyes, subjects felt dry just after the change of $\mathrm{RH}$, but after $90 \mathrm{~min}$ they did not feel dry, suggesting that they grew acclimatized as time passed. Accordingly, there was no coincidence between physiological measurement and subjective assessment in the skin and the eyes. On the contrary, subjects did not feel dry in the nose and throat just after the change of humidity, but after $90 \mathrm{~min}$, they felt dry under low RH (Fig. 10), showing a coincidence with the physiological measurement. It is thought that humans seem to feel dryness in the nose and throat more easily under low RH. Measurement of the frequency of blinking was not conducted just after moving to the test room, but based on the result of subjective assessment, we expect that physiological responses concerning the dryness of the body parts that are exposed to the environment for a long time, such as the eyes and skin, are sensitive to changes in humidity and respond rapidly to such changes. On the contrary, for dryness of the nose and the throat, SCT increased only under $10 \% \mathrm{RH}$, and subjects felt dry only $90 \mathrm{~min}$ after moving to the test room without any feeling of dryness just after moving, suggesting that they had a slower reaction to the change of humidity than in the eyes and the skin. This result may be due to the humidifying function of the nose and the fact that the nose and the throat are covered with mucous membrane and not exposed to the outer environment directly. The lack of direct exposure is especially relevant in the case of the nasal cavity, which has long ductal morphologic characteristics and the functional characteristics of regulating the temperature and humidity of inspired air. As mentioned previously, the nasal cavity cilia play an important role in the self-defense mechanism by protecting against foreign body infiltration and preventing viral infection. Therefore, these are thought to function well in $30 \%$ $\mathrm{RH}$ for short periods. In practical life, we are exposed to humidity levels below 10\% RH when facing warm air blown directly from a heater. In this study, we evaluated the effect of low RH by measuring SCT after 90 min. However, based on the response of the activity of the nasal cavity cilia in this experiment, we can expect that their function will deteriorate if one is exposed to low $\mathrm{RH}$ for a long time, even a $30 \% \mathrm{RH}$ environment. Thus, further evaluation on the physiological effects of prolonged exposure to various $\mathrm{RH}$ levels is recommended. It is not uncommon that the humidity in indoor environments in winter drops to lower than $30 \% \mathrm{RH}$ due to the heating system. For that reason, skin diseases resulting from dryness of the skin, feelings of fatigue and stress due to dryness of the eyes, and influenza and respiratory diseases due to dryness of the nasal mucosa could be harmful to human health in the low $\mathrm{RH}$ of indoor environments in winter. According to the subjective evaluation, subjects felt cool immediately at the trunk, legs and throughout the whole body after RH was changed. However, subjects only slightly perceived dryness of the nose, throat, face and hands as a result of the change of humidity, and feeling this dryness took some time. As a result, the sense of dryness is not as sensitive as that of temperature, and it is difficult for subjects to become aware of the change of humidity.

In conclusion, there was no effect of $\mathrm{RH}$ on the activity of the sebaceous gland and on cardiovascular reactions like blood pressure and HR. However, it was obvious that low RH affects SCT, causes dryness of the ocular mucosa and the stratum corneum of the skin, and causes a decrease in mean skin temperature. Namely, the eye and skin become dry in $30 \% \mathrm{RH}$, the nasal cavity becomes dry as well as the eye and skin in $10 \% \mathrm{RH}$, and the mean skin temperature decreases in $10 \% \mathrm{RH}$. These findings suggested that to avoid dryness of the eyes and skin, an RH higher than $30 \%$ must be maintained, and to avoid dryness of nasal mucous membrane, an RH higher than $10 \%$ must be maintained. Subjects felt cool immediately after the change of $\mathrm{RH}$, while they perceived dryness only slightly at the change of humidity. Therefore, not only the temperature but also the humidity should be checked using a thermohygrometer in heated indoor areas in the winter season.

Acknowledgements The authors would like to thank the students who participated in the experiment as subjects. The measurement of mucociliary activity using saccharin was conducted under the instruction of Prof. Dr. Yuichi Majima at the Dept. of Otorhinolaryngology, School of Medicine, Mie University. This study was supported by a Grant-in-Aid for the 21st Century COE program, a Grant-in-Aid for Scientific Research (No.15107005) from the Japan Society for the Promotion of Science, and funding from Techno Ryowa Ltd.

\section{References}

Andrasco G, Schoessler JP (1980) The effect of humidity on the dehydration of soft contact lenses on the eye. Int Cont Lens Clin 7: 210-212

Cook TH, Craft TJ (1958) Topographics of skin, non-dry skin, and cosmetically treated dry skin as quantified by skin profilometry. J Soc Cos Chem 36: 143-152

Eng WG, Harada LK, Jagerman LS (1982) The wearing of hydrophilic contact lenses aboard a commercial jet aircraft. I. Humidity effects on fit. Aviat Space Environ Med 53: 235-238

Fanger PO (1972) Thermal comfort. McGraw-Hill, New York Fujimoto S, Watanabe T (1969) Studies on the body surface area of Japanese. Acta Med Nagasaki 13: 1-13

Flynn WJ, Miller II RE, Tredici TJ, Block MG (1988) Soft contact lens wear at altitude: effect of hypoxia. Aviat Space Environ Med 59: 44-48

Gaul E, Underwood GB (1952) Reation of dewpoint and barometric pressure to chapping of normal skin. J Int Dermatol 19: 9-19

Grice K, Sattar H, Baker H (1972) The effect of ambient humidity on transepidermal water loss. J Int Dermatol 58: 343-346

Hamano H (1982) Physiology of the cornea-studies on the precorneal tear film. Jpn J Contact Lens 24: 18-25 [In 


\section{Japanese]}

Harper GJ (1961) Airborne micro-organisms: survival tests with four viruses. J Hyg Camb 59: 479-486

Harper GJ (1963) The influence of environment on the survival of airborne virus particles in the laboratory. Arch Ges Virusforsch 13: 64-71

King DC, Michael KM (1957) Muscular tension and the human blink rate. J Exp Psychol 53: 113-116

Satsuta K, Noriki H, Sakai F, Yabuuchi K (1985) Studies on the chronological difference in influenza epidemics: with special reference to average atmospheric temperature and average relative humidity. J Infect Chemother 59: 355-365 [In Japanese]

Koshimizu H, Tochihara Y, Ikeda K (2002) Changes in thermal comfort and subjective symptoms of factory workers by the use of humidifiers in low-humidity rooms in winter. J Archit Plann Environ Eng, AIJ, 552: 9-14 [In Japanese]

Lale AM, Mason JDT, Jones NS (1998) Mucociliary transport and its assessment: a review. Clin Otolaryngol 23: 388-396

Maehara N, Watanabe A, Kurosawa S (1991) Physiological and psychological effects of low humidity and low air pressure environment in passenger aircrafts. J Science Labour 67: 275-292 [In Japanese]

Nilsson SEG, Andersson L (1986) Contact lens wear in dry environments. Acta Ophthalmologica 64: 221-225

Ogawa H (1999) Law for maintenance of sanitation in buildings. Building Management Education Center, Tokyo, $15-17$

Ponder E, Kennedy WP (1928) On the act of blinking. Quarterly J Exp Physiol 18: 89-110

Rankin N (1998) What is optimum humidity? Respir Care Clin N Am 4: 321-328

Sakakura Y, Ukai K, Majima Y, Murai S, Harada T, Miyoshi Y (1983) Nasal mucociliary clearance under various conditions. Acta Otolaryngol 96: 167-173
Satsuta K, Noriki H, Sakai F, Yabuuchi K (1985) Studies on the chronological difference in influenza epidemics-with special reference to average atmospheric temperature and average relative humidity. Kansenshogaku Zasshi 59: 355-365 [In Japanese]

Stern JA, Walrath LC, Goldstein R (1984) The endogenous eyeblink. Psychophysiol 21: 22-33

Tada H (1986) Eyeblink rates as a function of the interest value of video stimuli. Tohoku Psychologica Folia 45: 107-113

Watanabe A, Kimotsuki K, Shinichiro I, Takashi Y, Toyohiko M, Fusajiro M, Toyonaga A (1977) Effect of humidity on the physiological and psychological responses under mild ambient temperature (Rep.2). J Sci Labour 53: 199-219 [In Japanese]

Williams R, Rankin N, Smith T, Galler D, Seakins P (1996) Relationship between the humidity and temperature of inspired gas and the function of the airway mucosa. Crit Care Med 24: 1920-1929

Yoshikuni K (1984) Assessment of the function of skin surface lipids influencing the water content of the stratum corneum. Nippon Hifuka Gakkai Zasshi 94: 1253-1258 [In Japanese]

Yoshikuni K, Tagami H, Inoue K, Yamada M (1985) Evaluation of the influence of ambient temperature and humidity on the hydration level of the stratum corneum. Nippon Hifuka Gakkai Zasshi 95: 591-595 [In Japanese]

Received: May 10, 2005

Accepted: October 4, 2005

Correspondence to: Yujin Sunwoo, Department of Ergonomics, Kyushu Institute of Design, 4-9-1 Shiobaru, Minami-ku, Fukuoka 815-8540, Japan

Phone: +81-92-553-4307

Fax: +81-92-553-4302

e-mail: sunwooyujin@yahoo.co.jp 